\title{
Water promoted catalyst-free anti-Markovnikov addition of thiols to styrenes
}

\author{
Barahman Movassagh ${ }^{*}$ and Mozhgan Navidi \\ Department of Chemistry, K. N. Toosi University of Technology, \\ P.O. Box 16315-1618, Tehran-Iran \\ E-mail: bmovass1178@yahoo.com
}

\begin{abstract}
A facile and convenient catalyst-free method for the anti-Markovnikov addition of thiols to styrenes at room temperature in water has been investigated.
\end{abstract}

Keywords: Anti-Markovnikov addition, thiols, water, styrenes

\section{Introduction}

Sulfur-containing compounds are found in a number of natural products and are of particular importance regarding pharmaceutically active substances. ${ }^{1}$ Nucleophilic addition reactions of thiols to electron-deficient olefins to form a carbon-sulfur bond constitutes a key reaction in biosynthesis as well as in the synthesis of biologically active compounds such as the calcium antagonist diltiazem. ${ }^{2}$ The addition of thiols onto carbon-carbon double bonds is either catalyzed by protic $^{3}$ as well as Lewis acids ${ }^{4}$ or takes place through a free-radical mechanism. The reaction may proceed via an electrophilic pathway involving ionic processes or a free-radical-chain pathway. ${ }^{5}$ Free-radical pathway has been well studied, ${ }^{6}$ but little work exists on the electrophilic pathways. However, the addition of thiols to styrene substrates has not been adequately studied, probably because they polymerize so easily under acidic conditions. ${ }^{4}$ Therefore, addition of thiols to styrenes has been studied with mild catalysts. ${ }^{7}$ Although the Markovnikov additions of thiols to styrenes are common, ${ }^{3,4,7 \mathrm{c}}$ additions leading to anti-Markovnikov products are rare. ${ }^{7 \mathrm{a}, 7 \mathrm{c}}$ There are, also, several catalyzed addition of organic disulfides to styrenes in literature. ${ }^{8-10}$ Unfortunately, various disadvantages such as long reaction times, low to moderate yields, use of toxic solvents, and use of costly and/or difficultly available catalysts encountered in some of the reported procedures necessitate the development of a more efficient and convenient method.

The organic reactions in aqueous media have attracted considerable attention in recent years, since they offer a powerful tool for minimizing waste production and harmful organic solvent dispersal. ${ }^{11}$ Some properties of water make this solvent very attractive (i.e. non-toxicity, non- 
inflammability, high heat capacity, possibility of controlling $\mathrm{pH}$, isolating insoluble solid products by filtration and recycling inorganic catalysts and water itself), allowing organic processes in aqueous medium to be safer, very efficient, and highly selective. ${ }^{12}$ The high efficiency and selectivity, and ease of product isolation prompted us to investigate its use for addition of thiols to styrenic substrates.

\section{Results and Discussion}

In our previous studies, ${ }^{13,14}$ we investigated the Michael additions of thiols and thiolate anions, generated from reductive cleavage of disulfides, to $\alpha, \beta$-unsaturated carbonyl compounds under neat conditions and $\mathrm{Zn} / \mathrm{AlCl}_{3}$-mediated in aqueous media. We report here an efficient synthesis of sulfides via the anti-Markovnikov addition of thiols to olefins in water at $25{ }^{\circ} \mathrm{C}$ in the absence of any catalyst (Scheme 1).

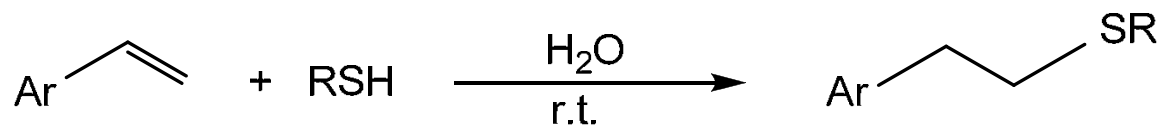

\section{Scheme 1}

In a model reaction, styrene $(1 \mathrm{mmol})$ was taken as a representative olefin; this was treated, with vigorous stirring, with thiophenol $(1.2 \mathrm{mmol})$ in the absence of a catalyst in metal-free water at room temperature under aerial conditions; after $5 \mathrm{~min}$, as monitored by TLC, the isolated yield of the product was $98 \%$. Under the same reaction conditions, another experiment was also carried out under argon; the addition reaction still proceeded to afford the antiMarkovnikov product within $5 \mathrm{~min}$ in $96 \%$ yield. The reaction was also conducted, under the same reaction conditions, in various solvents affording low to good yields of the product (Table 1).

To establish the generality of this process, various olefins were treated with a range of thiols in water at room temperature under catalyst-free and aerial conditions. Table 2 shows the results of the regioselective addition of thiols to various olefins under catalyst-free conditions. We found that this method was applicable for the preparation of sulfides from the reaction of aromatic thiols with styrenes of the type $\mathrm{ArCH}=\mathrm{CH}_{2}$ (entries 1-13, Table 2); these reactions are in general very fast (4-17 $\mathrm{min}$ ), clean and high yielding (73-98\%). Surprisingly, 1,1-diphenylethylene, styrene of type $\mathrm{Ph}_{2} \mathrm{C}=\mathrm{CH}_{2}$ (entry 14, Table 2) appears to be less reactive than the previously mentioned ones. In the latter case longer reaction time (360 $\mathrm{min}$ ) had to be employed in order to obtain the corresponding sulfide in satisfactory yield. However, under the same conditions, aliphatic olefins (entries 15 and 16, Table 2) did not produce the expected sulfides after prolonged reaction times and even higher temperature. The reaction was also conducted with two non-styrenic olefins such as 1-octene and 1-heptene (entries 17 and 18, Table 2); in both cases, 
no reaction took place with thiophenol at room temperature after $24 \mathrm{~h}$, but considerable amounts of the anti-Markovnokov products (73\% and 54\% respectively) were formed in $60-90$ min.

\section{Conclusions}

In conclusion, we have established a facile catalyst-free method for the anti-Markovnikov addition of thiols to a variety of olefinic compounds. In this respect, the highly regioselective addition of aromatic thiols to styrenes are very fast giving the products in high to excellent yields. This method is endowed with several unique merits, namely, simplicity in operation, mild reaction conditions, avoiding hazardous organic solvents, toxic and expensive reagents, short reaction times, and high product yields. This environmentally benign process represents a suitable option to existing methods.

Table 1. Reaction of styrene with thiophenol in various solvents ${ }^{\mathrm{a}}$

\begin{tabular}{ccc}
\hline Entry & Solvent & ${\text { Yields }(\%)^{\mathrm{b}}}^{\mathrm{M}}$ \\
\hline 1 & $\mathrm{MeOH}$ & 64 \\
2 & $\mathrm{MeCN}$ & 77 \\
3 & $n-\mathrm{Hexane}$ & 66 \\
4 & $\mathrm{CHCl}_{3}$ & 54 \\
5 & $\mathrm{PhMe}$ & 23 \\
6 & $\mathrm{THF}$ & 56 \\
7 & Neat & 72 \\
\hline
\end{tabular}

${ }^{\mathrm{a}}$ Conditions: styrene ( 1 equiv) was treated with thiophenol (1.2 equiv) at r.t. for 5 min. ${ }^{\mathrm{b}}$ Isolated yield.

Table 2. Regioselective addition of thiols to olefins in water and catalyst-free conditions

\begin{tabular}{llccc}
\hline Entry & Alkene & Thiol & Product & $\begin{array}{c}\text { Condition } \\
(\%)^{\mathrm{a}, \mathrm{b}}\end{array}$ \\
\hline 1 & $2{ }^{\circ} \mathrm{C} ; 5 \mathrm{~min}$ & $98^{7 \mathrm{c}}$
\end{tabular}


Table 2. Continued

\begin{tabular}{|c|c|c|c|c|c|}
\hline Entry & Alkene & Thiol & Product & Condition & $\begin{array}{l}\text { Yields } \\
(\%)^{a, b}\end{array}$ \\
\hline 4 & & $\mathrm{PhSH}$ & & $25^{\circ} \mathrm{C} ; 5 \mathrm{~min}$ & $90^{10}$ \\
\hline 5 & & & & $25^{\circ} \mathrm{C} ; 10 \mathrm{~min}$ & $80^{10}$ \\
\hline 6 & & $\mathrm{PhSH}$ & & $25^{\circ} \mathrm{C} ; 5 \mathrm{~min}$ & $84^{10}$ \\
\hline 7 & & & & $25^{\circ} \mathrm{C} ; 5 \mathrm{~min}$ & $80^{15}$ \\
\hline 8 & & & & $25^{\circ} \mathrm{C} ; 8 \mathrm{~min}$ & $77^{15}$ \\
\hline 9 & & 3 & & $25^{\circ} \mathrm{C} ; 10 \mathrm{~min}$ & $74^{15}$ \\
\hline 10 & & $\mathrm{eO}^{-}$ & & $25^{\circ} \mathrm{C} ; 7 \mathrm{~min}$ & $80^{15}$ \\
\hline 11 & & $\mathrm{PhSH}$ & & $25^{\circ} \mathrm{C} ; 10 \mathrm{~min}$ & 87 \\
\hline 12 & & & & $25{ }^{\circ} \mathrm{C} ; 15 \mathrm{~min}$ & $81^{10}$ \\
\hline 13 & & $\mathrm{PhSH}$ & & $25^{\circ} \mathrm{C} ; 17 \mathrm{~min}$ & $73^{16}$ \\
\hline 14 & $\mathrm{Ph}_{2} \mathrm{C}=\mathrm{CH}_{2}$ & $\mathrm{PhSH}$ & $\mathrm{h}_{2} \mathrm{CHCH}_{2} \mathrm{~S}$ & $\begin{array}{c}25^{\circ} \mathrm{C} ; 360 \\
\min \end{array}$ & $75^{17}$ \\
\hline 15 & & $\left(\mathrm{CH}_{3}\right)_{3} \mathrm{CSH}$ & N.R. & $25^{\circ} \mathrm{C} ; 24 \mathrm{~h}$ & - \\
\hline 16 & 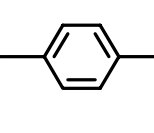 & $n-\mathrm{C}_{4} \mathrm{H}_{9} \mathrm{SH}$ & N.R. & $\begin{array}{l}30^{\circ} \mathrm{C}, 24 \mathrm{~h} \\
50^{\circ} \mathrm{C}, 24 \mathrm{~h}\end{array}$ & - \\
\hline
\end{tabular}


Table 2. Continued

\begin{tabular}{|c|c|c|c|c|c|}
\hline Entry & Alkene & Thiol & Product & Condition & $\begin{array}{l}\text { Yields } \\
(\%)^{\mathrm{a}, \mathrm{b}}\end{array}$ \\
\hline 17 & $\mathrm{CH}_{3}\left(\mathrm{CH}_{2}\right)_{5} \mathrm{CH}=\mathrm{CH}_{2}$ & $\mathrm{PhSH}$ & $\mathrm{CH}_{3}\left(\mathrm{CH}_{2}\right)_{6} \mathrm{CH}_{2} \mathrm{SPh}$ & $\begin{array}{c}30^{\circ} \mathrm{C}, 24 \mathrm{~h} \\
50^{\circ} \mathrm{C}, 1 \mathrm{~h}\end{array}$ & $\begin{array}{l}\text { N.R. } \\
73^{7 \mathrm{a}}\end{array}$ \\
\hline 18 & $\mathrm{CH}_{3}\left(\mathrm{CH}_{2}\right)_{4} \mathrm{CH}=\mathrm{CH}_{2}$ & $\mathrm{PhSH}$ & $\mathrm{CH}_{3}\left(\mathrm{CH}_{2}\right)_{5} \mathrm{CH}_{2} \mathrm{SPh}$ & $\begin{array}{l}30^{\circ} \mathrm{C}, 24 \mathrm{~h} \\
50^{\circ} \mathrm{C}, 1.5 \mathrm{~h}\end{array}$ & $\begin{array}{l}\text { N.R. } \\
54^{17}\end{array}$ \\
\hline
\end{tabular}

${ }^{a}$ Yields refer to pure isolated products. ${ }^{b}$ References for known compounds.

\section{Experimental Section}

General Procedures. Known products were characterized by comparison of their spectroscopic data with those of known samples. IR spactra were obtained using an ABB FTLA 2000 instrument. NMR spectra were recorded with either a Bruker AQS-300 or Bruker DRX-500 spectrometer with nominal frequencies of 300 and $500 \mathrm{MHz}$ for proton or 75 and $125 \mathrm{MHz}$ for carbon, respectively in $\mathrm{CDCl}_{3}$ solutions.

\section{Representative experimental procedure for preparation of 1-(2-phenylsulfanylethyl) benzene (entry 1, Table 1$)^{7 \mathrm{c}}$}

In a typical experiment, a mixture of styrene (104 mg, $1 \mathrm{mmol})$, thiophenol (132 mg, $1.2 \mathrm{mmol})$, and water $(2 \mathrm{~mL})$ was placed in a test tube. The reaction mixture was magnetically stirred at room temperature $\left(25^{\circ} \mathrm{C}\right)$ for $5 \mathrm{~min}$. The course of the reaction was monitored by TLC until the starting materials had completely disappeared. Then, the organic materials were extracted with chloroform $(2 \times 5 \mathrm{~mL})$. The organic layer was washed with $10 \%$ sodium hydroxide solution $(2 \times$ $5 \mathrm{~mL})$ and water $(5 \mathrm{~mL})$. Drying $\left(\mathrm{MgSO}_{4}\right)$ and evaporation of the organic layer gave the crude product, which was subjected to preparative TLC (silica gel, eluent $n$-hexane) to afford $209 \mathrm{mg}$ (98\%) of pure 1-(2-phenylsulfanylethyl) benzene as yellow oil; IR (neat) v 1437, 1473, $1586 \mathrm{~cm}^{-}$ 1. ${ }^{1} \mathrm{H}$ NMR (500 MHz) $\delta 3.00-3.03(\mathrm{~m}, 2 \mathrm{H}), 3.24-3.27$ (m, 2H), 7.25-7.32 (m, 4H), 7.36-7.40 (m, $4 \mathrm{H}), 7.43-7.46(\mathrm{~m}, 2 \mathrm{H}) ;{ }^{13} \mathrm{C} \mathrm{NMR}(125 \mathrm{MHz}) \delta 35.09,35.67,126.00,126.50,128.56,128.99$, $129.19,136.44,140.24$.

\section{Selected physical and spectral data}

1-[2-(4-Methoxyphenyl)sulfanylethyl] benzene (entry 3). Oil; IR (neat) v 1247, 1494, 1591 $\mathrm{cm}^{-1}$; ${ }^{1} \mathrm{H}$ NMR (500 MHz) $\delta$ 2.92-2.96 (m, 2H), 3.12-3.15 (m, 2H), $3.87(\mathrm{~s}, 3 \mathrm{H}), 6.92-6.95(\mathrm{~m}$, 2H), $7.23(\mathrm{~d}, J=7.2 \mathrm{~Hz}, 2 \mathrm{H}), 7.26-7.31(\mathrm{~m}, 1 \mathrm{H}), 7.35(\mathrm{t}, J=7.6 \mathrm{~Hz}, 2 \mathrm{H}), 7.43-7.46(\mathrm{~m}, 2 \mathrm{H}) ;{ }^{13} \mathrm{C}$ NMR $(125 \mathrm{MHz}) \delta 36.38,37.69,55.79,115.07,126.80,128.91,128.97,133.72,140.84,159.41$; 
MS (m/z) M 244 (97), 153 (100), 138 (23), 125 (7), 105 (35), 91 (9), 77 (22). Anal. Calcd for $\mathrm{C}_{15} \mathrm{H}_{16} \mathrm{OS}$ : C, 73.73; H, 6.60. Found: C, 74.06; H, 6.82.

1-(2-Phenylsulfanylethyl)-4-chlorobenzene (entry 4). ${ }^{10}$ Oil; IR (neat) v $1489,1586 \mathrm{~cm}^{-1} ;{ }^{1} \mathrm{H}$ NMR (300 MHz) $\delta 2.93-2.99(\mathrm{~m}, 2 \mathrm{H}), 3.18-3.24(\mathrm{~m}, 2 \mathrm{H}), 7.16-7.20(\mathrm{~m}, 2 \mathrm{H}), 7.25-7.46(\mathrm{~m}, 7 \mathrm{H})$; ${ }^{13} \mathrm{C}$ NMR $(75 \mathrm{MHz}) \delta 34.96,35.04,126.22,128.69,129.11,129.40,130.03,132.28,136.24$, 138.68 .

1-[1-Methyl-2-(4-methylphenyl)sulfanylethyl] benzene (entry 9). ${ }^{15}$ Oil; IR (neat) v 1509, $1612 \mathrm{~cm}^{-1}$; ${ }^{1} \mathrm{H}$ NMR $(300 \mathrm{MHz}) \delta 1.49$ (d, $\left.J=6.6 \mathrm{~Hz}, 3 \mathrm{H}\right), 2.42$ (s, 3H), 3.03-3.15 (m, 2H), 3.29 $(\mathrm{dd}, J=12.0,5.3 \mathrm{~Hz}, 1 \mathrm{H}), 7.19(\mathrm{~d}, J=8.3 \mathrm{~Hz}, 2 \mathrm{H}), 7.26-7.44(\mathrm{~m}, 6 \mathrm{H}) ;{ }^{13} \mathrm{C} \mathrm{NMR}(75 \mathrm{MHz}) \delta$ 21.05, 21.11, 39.53, 42.84, 126.62, 127.07, 128.60, 129.77, 130.02, 133.11, 136.03, 145.72.

1-(2-Phenylsulfanylethyl)-4-methoxybenzene (entry 11). Oil; IR (neat) v 1211, 1489, 1601 $\mathrm{cm}^{-1}$; ${ }^{1} \mathrm{H}$ NMR $(500 \mathrm{MHz}) \delta$ 2.93-2.96 (m, 2H), 3.19-3.22 (m, 2H), $3.85(\mathrm{~s}, 3 \mathrm{H}), 6.90-6.92(\mathrm{~m}$, 2H), $7.18(\mathrm{~d}, J=8.5 \mathrm{~Hz}, 2 \mathrm{H}), 7.25(\mathrm{t}, J=7.3 \mathrm{~Hz}, 1 \mathrm{H}), 7.36(\mathrm{t}, J=7.9 \mathrm{~Hz}, 2 \mathrm{H}), 7.42(\mathrm{~d}, J=7.6$ $\mathrm{Hz}, 2 \mathrm{H}) ;{ }^{13} \mathrm{C}$ NMR $(125 \mathrm{MHz}) \delta 35.19,35.82,55.71,114.38,126.38,129.37,129.62,129.93$, 132.77, 136.95, 158.70; MS (m/z) M+ 244 (95), 153 (100), 138 (23), 125 (7), 105 (36), 91 (10), 77 (21). Anal. Calcd for $\mathrm{C}_{15} \mathrm{H}_{16} \mathrm{OS}$ : C, 73.73; H, 6.60. Found: C, 73.56; H, 6.62.

1-(1-Phenyl-2-phenylsulfanylethyl) benzene (entry 14). ${ }^{17} \mathrm{Oil}$; IR (neat) v 1494, $1586 \mathrm{~cm}^{-1} ;{ }^{1} \mathrm{H}$ NMR $(300 \mathrm{MHz}) \delta 3.80(\mathrm{~d}, J=7.8 \mathrm{~Hz}, 2 \mathrm{H}), 4.44(\mathrm{t}, J=7.8 \mathrm{~Hz}, 1 \mathrm{H}), 7.33-7.54(\mathrm{~m}, 15 \mathrm{H}) ;{ }^{13} \mathrm{C}$ NMR $(75 \mathrm{MHz}) \delta 39.79,50.77,126.25,126.95,128.18,128.81,129.18,129.57,136.85,143.28$.

\section{Acknowledgements}

We thank the K. N. Toosi University of Technology Research Council and Iranian National Science Foundation (INSF, Grant No. 86063/21) for their financial support.

\section{References}

1. Page, P. C. B. Organo-Sulfur Chemistry I \& II, Springer: Berlin, 1999.

2. (a) Clark, J. H. Chem. Rev. 1980, 80, 429. (b) Trost, B. M.; Keely, D. E. J. Org. Chem. 1975, 40, 2013. (c) Nishimura, K.; Ono, M.; Nagaoka, Y.; Tomioka, K. J. Am. Chem. Soc. 1997, 119, 12974.

3. (a) Screttas, C. G.; Micha-Screttas, M. J. Org. Chem. 1979, 44, 713. (b) Wolf, F.; Finke, H. Z. Chem. 1972, 12, 180. (c) Ipatieff, V. N.; Pines, H.; Friedman, B. S. J. Am. Chem. Soc. 1938, 60, 2731.

4. (a) Mukaiyama, T.; Izawa, T.; Saigo, K.; Takai, H. Chem. Lett. 1976, 355. (b) Belly, M.; Zamboni, R. J. Org. Chem. 1989, 54, 1230.

5. Curran, D. P. In Comprehensive Organic Synthesis; Trost, B. M.; Fleming, I., Ed.; Vol. 4, Pergamon: New York, 1991, pp 715-777. 
6. Griesbaun, K. Angew. Chem., Int. Ed. 1970, 9, 273.

7. (a) Kumar, P.; Pandy, R. K.; Hegde, V. R. Synlett 1999, 1921. (b) Braga, A. L.; Silveira, C. C.; Dornelles, L.; Zeni, G.; Galarza, F. A. D.; Wessjohann, L. A. Synth. Commun. 1995, 25, 3155. (c) Kanagasabapathy, S.; Sudalai, A.; Benicewicz, B. C. Tetrahedron Lett. 2001, 42, 3791.

8. Taniguchi, N. J. Org. Chem. 2006, 71, 7874.

9. Takeuchi, M.; Shimakoshi, H.: Kano, K. Organometallics 1994, 13, 1208.

10. Ranu, B. C.; Mandal, T. Tetrahedron Lett. 2006, 47, 6911.

11. (a) Tundo, P.; Anastas, P. T. Green Chemistry: Challenging Perspectives, Oxford University Press: Oxford, 1999. (b) DeSimone, J. M. Science 2002, 297, 799.

12. Li, C. J.; Chang, T. H. Organic Reactions in Aqueous Media, Wiley: New York, 1997.

13. Movassagh, B.; Shaygan, P. Arkivoc 2006, (xii), 130.

14. Movassagh, B.; Zakinezhad, Y. Z. Naturforsch. 2006, 61b, 47.

15. Ito, O.; Matsuda, M. J. Org. Chem. 1982, 47, 2261.

16. Church, D. F.; Gleicher, G. J. J. Org. Chem. 1975, 40, 536.

17. Screttas, C. G.; Micha-Screttas, M. J. Org. Chem. 1978, 43, 1064. 